In beiden Metallen sind also die Defektelektronen trotz des negativen Vorzeichens der Hall-Konstanten stark am elektrischen Leitungsvorgang beteiligt, aber der Einfluß der $s$-Elektronen ist überwiegend $\left(\varkappa_{\mathrm{s}} / \varkappa>50 \%\right)$.

Vergrößert man also die Anzahl der Defektelektronen, indem man zu Rh oder Ir übergeht, wo je ein Elektron weniger vorhanden ist, als zur vollständigen Auffüllung des inneren Bandes (d-Bandes) notwendig wäre, so schließt man aus dem positiven Vorzeichen von $A_{\mathrm{H}}$ auf die Ungleichung $x_{\mathrm{s}} / x_{\mathrm{d}}<\sqrt{n_{\mathrm{s}} / n_{\mathrm{d}}}$. Da man $n_{\mathrm{s}}$ ind $n_{\mathrm{d}}$ in diesen Fällen nicht kennt, gelingt

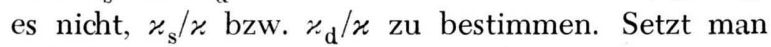
jedoch versuchsweise $n_{\mathrm{s}}=0=\varkappa_{\mathrm{s}} / \varkappa$ und $n_{\mathrm{d}}=1$, so erhält man nach (1) für Rh $10^{6} \cdot A_{\mathrm{H}}=+855$ und Ir $10^{6} \cdot A_{\mathrm{H}}=+895$, also viel zu große positive Werte für $A_{H}$. Dies läßt darauf schließen; daß auch bei diesen Metallen noch merklich s-Elektronen am Leitungsvorgang beteiligt sind, die den Absolutwert von $A_{\mathrm{H}}$ verkleinern.

Was schließlich den Fall des Ru betrifft, so ist dessen große positive Hall-Konstante nicht ohne weiteres verständlich, weil Gl. (1) selbst im Extremfall völlig ausfallender s-Elektronen $\left(n_{\mathrm{s}}=0\right.$ oder $\left.n_{\mathrm{s}}=2\right)$ nur $10^{6} \cdot A_{\mathrm{H}}=+425$ mit $n_{\mathrm{d}}=2$ (nach Tab. 1) ergeben würde. Eine mögliche Erklärung sehen wir darin, daß das s-Band fast voll besetzt ist, so daß $n_{\mathrm{s}}^{*}=2-n_{\mathrm{s}}$ gilt, wo $n_{\mathrm{s}}^{*}<1$ die Anzahl der Löcher im s-Band bedeutet, die nunmehr positive Beiträge zum Hall-Effekt liefern. Demgemäß muß Gl. (1) jetzt modifiziert werden $\mathrm{zu}$

$$
A_{\mathrm{H}}=+\frac{c}{e N}\left[\left(\frac{\varkappa_{\mathrm{s}}}{\varkappa}\right)^{2} \frac{1}{n_{\mathrm{s}}^{*}}+\left(\frac{\varkappa_{\mathrm{d}}}{\varkappa}\right)^{2} \frac{1}{n_{\mathrm{d}}}\right] \ldots
$$

Diese Deutung wird gestützt durch unseren Befund $^{7}$ an Re, das eine volle Besetzung des s-Niveaus im freien Atom (vgl. Tab. 1) mit der extrem hohen Hall-Konstante $10^{6} \cdot A_{\mathrm{H}}=+3150$ verbindet. Eine andere Erklärungsmöglichkeit bestände in der Berücksichtigung einer feineren Struktur des d-Bandes, entsprechend der Aufspaltung des d-Niveaus der freien-Atome in 5 Teilniveaus zu je zwei d-Elektronen.

Wir danken wiederum der Braunschweigischen Wissenschaftlichen Gesellschaft für die finanzielle Unterstützung, durch die der große technische Aufwand, der für diese Messungen notwendig ist, ermöglicht wurde.

\title{
Die Fermi'sche Grenzenergie bei den elektronischen Halbleitern
}

\section{Von Helmut Müser}

Aus dem Physikalischen Institut der Universität Frankfurt a. M.

(Z. Naturforschg. 5 a, 18-25 [1950]; eingegangen am 19. Februar 1949)

Die Vorstellungen des Wilsonschen Halbleitermodells werden quantitativ durchgeführt und die Abhängigkeit der Fermischen Grenzenergie von Temperatur, Konzentration und Art der Störstellen berechnet. Es ergibt sich, daß die Grenzenergie das elektrische Verhalten des Halbleiters entscheidet. Bisher ungeklärte experimentelle Ergebnisse werden gedeutet.

$\mathrm{B}$ eim metallischen Leiter liegt die Fermische Grenzenergie (६) innerhalb, beim Halbleiter außerhalb der erlaubten Energiebänder oder unmittelbar an ihrem Rande.

Die Leitfähigkeit der Metalle ist klassisch bestimmt durch die Anzahl der freien Elektronen und ihre Beweglichkeit. Im quantenmechanischen Bild darf man, wie Fröhlich ${ }^{1}$ gezeigt hat, in übertragenem Sinne noch von einer Zahl der freien Elektronen pro Volumeneinheit sprechen: sie ist $N_{\mathrm{F}}={ }^{4 / 3} D(\zeta) E_{\mathrm{tr}}(\zeta)$. Dabei bedeutet $D(\zeta)$ die Zahl der Elektronenterme pro Energie- und Volumeinheit in der Nähe der

${ }^{1} \mathrm{H}$. Fröhlich, Proc. Cambridge philos. Soc. 31, 277 [1935].
Grenzenergie und $E_{\text {tr }}$ die Translationsenergie eines Elektrons mit der Energie $\zeta$.

Das Hauptproblem in der Elektronentheorie der Metalle ist die Bestimmung der Beweglichkeit oder der freien Weglänge der Elektronen, weil diese durch Temperatur, Verunreinigungen usw. stärker beeinflußt wird als die Zahl der freien Elektronen. $\zeta$ pflegt man näherungsweise durch $\zeta_{0}$, die Grenzenergie beim absoluten Nullpunkt, zu ersetzen. Tatsächlich ist diese Näherung für die Metalle ausreichend, da einmal eine kleine Änderung von : die Konzentration der freien Elektronen nur wenig ändert ${ }^{2}$, zum andern

2 Etwa mit $\zeta^{\mathbf{3} / 2}$, wenn $\zeta$ vom Bandrand an gerechnet wird. 
wird in Gl. (4) gezeigt, daß $\zeta$ exakt temperaturunabhängig ist, wenn sich die Energieterme symmetrisch um $\zeta$ verteilen. Das ist aber bei den Metallen für gewöhnlich angenähert der Fall.

Die Verteilung der Elektronenterme wird bestimmt durch die Funktion $D(E)$. Wenn sich die Elektronen in dem periodischen Potential eines Kristallgitters bewegen, so sind ihnen gewisse Energiewerte ,verboten“, andere „,erlaubt“. In den verbotenen Bändern ist $D=0$, in den erlaubten hat es einen endlichen Wert.

Die Besetzung der Terme regelt die Fermi-Statistik: die Wahrscheinlichkeit, einen bestimmten Platz besetzt zu finden, ist

$$
f(E)=\frac{1}{1+e^{(E-\zeta) / k T}} .
$$

Es gibt danach $[g \cdot D(E) \cdot f(E) \cdot d E]$ Elektronen mit einer Energie zwischen $E$ und $E+d E$. g bedeutet das Gewicht des Terms. Für die Quantenzustände des Kristalls ist wegen des Elektronenspins $g=2$.

Die Gesamtzahl der Elektronen je $\mathrm{cm}^{3}$ ist konstant:

$$
N=\int_{0}^{\infty} g \cdot D(E) \cdot f(E) \cdot d E .
$$

Differenziert man die Beziehung (2) nach der Temperatur, so sieht man, daß $\zeta$ temperaturunabhängig ist, wenn die Terme um $\zeta$ symmetrisch verteilt sind. Man erhält nämlich nach Einsetzen von $f(E)$ aus (1):

$\int_{0}^{\infty} \frac{D(E)}{(50)^{2}(E-\zeta) / 2 k T}\left(\frac{E-\zeta}{T}+\frac{d \zeta}{d T}\right) d E=0$.

Wegen des $\mathfrak{C V}^{2}{ }^{2}$ im Nenner liefern alle Energiewerte $E$, die mehrere $k T$ von $\zeta$ entfernt liegen, keinen Beitrag zu dem Integral. Ist $D(E)$ für $E \approx \zeta$ symmetrisch um $\zeta$, so steht unter dem Integral nach Multiplizieren mit $(E-\zeta)$ eine in bezug auf $\zeta$ ungerade Funktion, deren Integral verschwindet. Also wird, da $D(E)$ nur positive Werte annehmen kann:

$$
d \zeta / d T=0 .
$$

Ein Sonderfall liegt vor, wenn $D(E)=0$ für $E \approx \zeta$ ist. Dieser Fall ist typisch für die Halbleiter und wird im nächsten Abschnitt noch gesondert behandelt.

Bei den Halbleitern liegt $\zeta$ erheblich unterhalb des Leitungsbandes in einem Gebiet mit $D(E)=0$ oder besser $D(E) \approx 0$. Nennen wir $E_{0}$ die Energie des unteren Bandrandes, so ist $E_{0}-\xi \gg k T$. Die Terme des Leitungsbandes sind sehr dünn besetzt, die Elektronen sind also praktisch frei. Die Konzentration der freien Elektronen, die für das elektrische Verhalten des Halbleiters entscheidend ist, wird durch kleine Änderungen von $\zeta$ empfindlich verändert.

Übergangswiderstände an Korngrenzen, innere Sperrschichten u. ä. wären in dem Energieschema als Potentialberge aufzufassen, deren Übersteigen den Elektronen erschwert ist. Es werden aber um so mehr Elektronen einen solchen Wall überschreiten können, je mehr im obersten Band vorhanden sind. Auch die Leitfähigkeit eines Körpers mit Korngrenzenwiderständen wird also mit der Konzentration der freien Elektronen zunehmen und damit durch die Lage der Fermischen Grenzenergie bestimmt werden.

1. Die Störstellenhypothese und ihre Folgerungen fürdie Grenzenergie

In dem ursprünglichen Wilsonschen Halbleitermodell ${ }^{3}$ lag $\zeta$ in der Mitte zwischen dem obersten besetzten (G-Band) und dem Leitungsband (F-Band). Das gilt nur in 1. Näherung. Wenn der Abstand zwischen F- und G-Band viele $k T$ groß ist und $\zeta$ mehrere $k T$ von jedem von beiden entfernt ist, so darf jedes Band durch ein einziges Niveau von der Energie seines Randes mit dem statistischen Gewicht $2\left(2 \pi m_{i}\right.$ $\left.k T / h^{2}\right)^{3 / 2}(i=\mathrm{F}, \mathrm{G})$ ersetzt werden ${ }^{4}$. Dabei bedeutet $m_{\mathrm{F}}$ die scheinbare Masse eines Elektrons im F-Band und $m_{\mathrm{G}}$ die scheinbare Masse eines Elektrons im G-Band. Die statistischen Gewichte der beiden Bänder verhalten sich also wie $m_{\mathrm{F}}{ }^{3 / 2}: m_{\mathrm{G}}{ }^{3 / 2}$. Damit die Anzahl der Löcher im G-Band gleich der Anzahl der Elektronen im F-Band ist, muß sein:

$$
\frac{\left(2 \pi m_{\mathrm{G}} k T / h^{2}\right)^{3 / 2}}{1+e^{\zeta / k T}}=\frac{\left(2 \pi m_{\mathrm{F}} k T / h^{2}\right)^{3 / 2}}{1+e^{(E-\zeta) / k T}} .
$$

Unter Vernachlässigung der 1 im Nenner wird also

$$
\zeta=\frac{E}{2}+\frac{3}{4} k \cdot T \ln \frac{m_{\mathrm{G}}}{m_{\mathrm{F}}},
$$

d. h. $\zeta$ wird linear abhängig von der Temperatur.

Die experimentellen Ergebnisse lassen sich aber mit diesem einfachen Modell nicht fassen. Wil s o $n^{5}$ bezog daher in seine Theorie die Störstellen ein, über deren Wirksamkeit durch die Versuche von Gudden und Pohl u. a. reichliches experimentelles

${ }^{3}$ A. H. Wils on, Proc. Roy. Soc [London], Ser. A 133,458 [1931].

${ }^{4}$ Vgl. Gl. (12).

5 A. H. Wils on, Proc. Roy. Soc. [London], Ser. A 134, 277 [1931]. 
Material vorlag. Eine Störstelle ist entweder eine Haftstelle (Fänger) oder ein Spender. Die Haftstelle ist charakterisiert durch das Auftreten eines zusätzlichen, örtlich festliegenden Terms in unserem Energieschema. Für einen Spender tritt außerdem zu der konstanten Anzahl der zur Verfügung stehenden Elektronen ein Elektron hinzu ${ }^{6}$. Durch Störstellen wird die Grenze $\zeta$ zwischen den Bändern verschoben, durch Spender nach oben und durch Fänger nach unten. Die Verschiebung ist temperaturabhängig; schon wenige Störterme genügen, sie zu bewirken, und erst damit wird eine Überschuß- oder Defektleitung mit so großen Hall-Effekten und Thermokräften, wie sie die Experimente ergeben, überhaupt verständlich.

Für die Temperaturabhängigkeit der Grenzenergie sind zwei Ursachen zu berücksichtigen: 1. Der Elektronenhaushalt kann durch Defekt oder Überschuß so gestört werden, daß die Grenzenergie beim absol. Nullpunkt an einer beliebigen Stelle zwischen den Bändern, nicht vorzugsweise in der Mitte liegt. 2. Im verbotenen Gebiet können Terme auftreten. Jeder dieser Fälle hat zur Folge, daß Gl. (4) nicht mehr gilt. Liegt $\zeta$ in der Nähe des Bandrandes, so wird die Verteilung der Terme auf der Energieskala nicht symmetrisch um $\zeta$ sein, da $D(E)$ nach dem Bandinnern zunimmt. Für das Gebiet zwischen den Bändern haben wir wegen der Störterme nun $D(E)$ $\approx 0 \mathrm{zu}$ schreiben. Liegt $\zeta$ mehrere $k T$ von den Bandrändern entfernt, so treten daher in dem Integral (3) zwei Beiträge in Konkurrenz: 1. Der Beitrag des Energiegebietes in der Umgebung von $\zeta$ (er ist klein wegen $D(E) \approx 0) ; 2$. der Beitrag des Energiegebietes an den Bandrändern; er ist ebenfalls klein wegen des großen $\mathrm{C}_{\mathrm{oj}}{ }^{2}$ im Nenner. Die beiden kleinen Beiträge konkurrieren so, daß bei niederen Temperaturen der Beitrag 1, bei hohen der Beitrag 2 ausschlaggebend wird. Der Übergang erfolgt bei einer um so höheren Temperatur, je dichter die Störterme in der Nähe der Grenzenergie sitzen und je weiter der nächste Bandrand entfernt ist.

Zur Veranschaulichung dieser Überlegungen zeigen die Abb. $1 \mathrm{a}-\mathrm{c}$ die Abhängigkeit der Grenzenergie von der Temperatur. Die Kurven werden zum besseren Verständnis vorweggenommen; die Berechnung geht aus dem 2. Abschnitt hervor.

Gewählt ist als Beispiel ein Modellhalbleiter, für den folgende Anuahmen gemacht werden:

${ }^{6}$ Diese allgemeine Formulierung für den Begriff der Störstellen findet sich bei J. H. G i s o lf, Ann. Physik 1, 1 [1947].

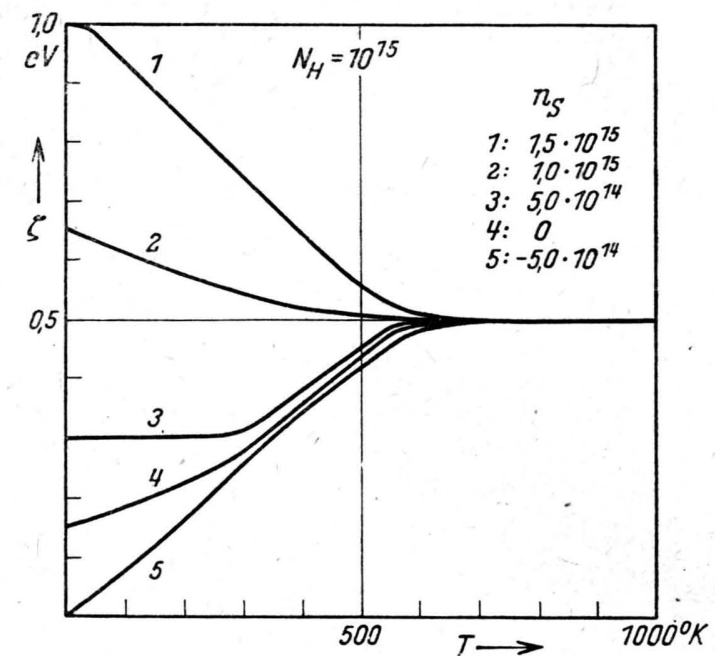

Abb. 1 a. Abhängigkeit der Grenzenergie von der Temperatur für einen Modellhalbleiter mit diskretem Haftniveau und schwacher Haftstellenkonzentration.

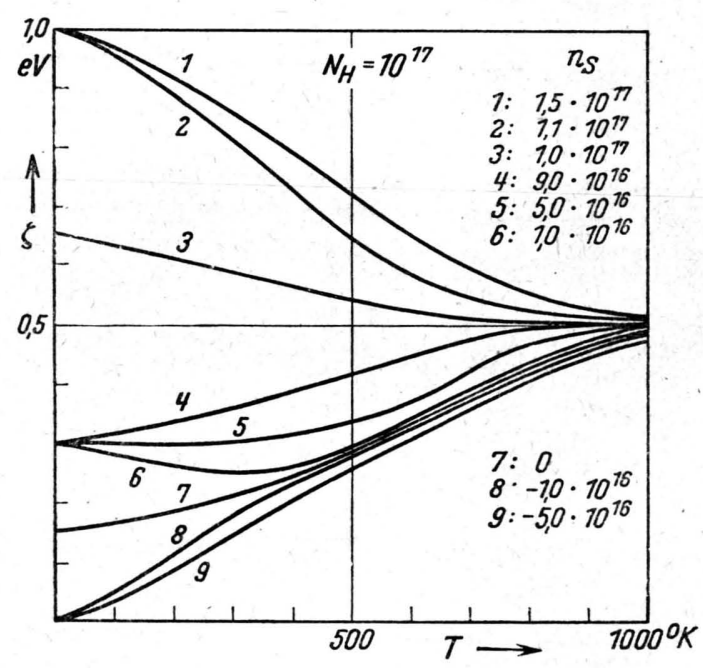

Abb. 1 b. Abhängigkeit der Grenzenergie von der Temperatur für einen Modellhalbleiter mit diskretem Haftniveau und starker Haftstellenkonzentration.

Abstand zwischen F- und G-Band: $1 \mathrm{eV}$;

Diskretes Störniveau (Haftstellenniveau) bei $0,3 \mathrm{eV}$ oberhalb des G-Bandes; (Abb. $1 \mathrm{a}$ und b);

Kontinuierlich zwischen 0 und $0,3 \mathrm{eV}$ verteilte $\mathrm{Haft}$ niveaus (Abb. 1c);

Konzentration der Haftstellen: $N_{\mathrm{H}}=10^{15}$ (Abb. 1 a), $10^{17}$ (Abb. 1 b), $10^{18}$ (Abb. 1c).

Variable Konzentration $n_{\mathrm{S}}$ der Störelektronen (Spenderelektronen).

Die Kurvenscharen zeigen für niedrige Temperaturen die gleiche Tendenz: Für die Änderung von $\zeta$ 


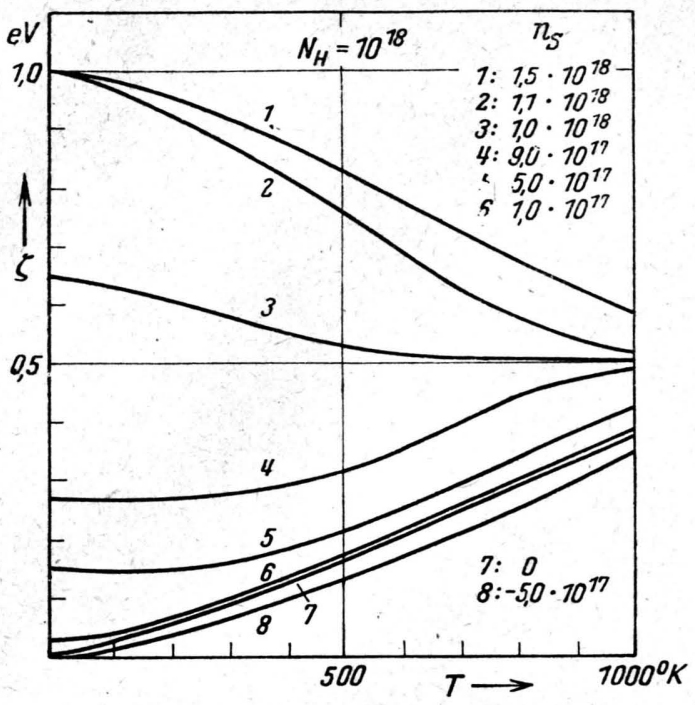

Abb. 1c. Abhängigkeit der Grenzenergie von der Temperatur für einen Modellhalbleiter mit kontinuierlicher Verteilung der Haftniveaus (Haftstellenkonzentration 10-mal größer als in Abb. 1 b).

sind die Terme in seiner Nähe maßgebend, entweder das nächstgelegene Band (Abb.1a), oder, wenn sie genügend konzentriert sind, die Störterme (Abb. $1 \mathrm{~b}$, besonders Kurve 4 und 6). Für diesen Teil der Kurve ist also die Verteilung der Störterme in der Nähe von $\zeta$ maßgebend. Von einer bestimmten Temperatur $\Theta$ ab (in Abb. 1 a etwa $250^{\circ} \mathrm{K}$, in Abb. 1 b etwa $400^{\circ} \mathrm{K}$, in Abb. $1 \mathrm{c} 600^{\circ} \mathrm{K}$ ) setzt sich der Einfluß der Bänder immer mehr durch, so daß $\zeta$ vom nächstgelegenen Band wegstrebt, bis es endlich konstant in der Mitte zwischen den Bändern liegt. Ein Halbleiter, der diesen Zustand erreicht hat, wird sich wie ein Eigenhalbleiter verhalten, so, wie es das ursprüngliche Wilsonsche Modell vorausgesagt hat. Für derartig hohe Temperaturen ist der Einfluß der Störstellen geschwunden. Die Temperatur, von der ab der Einfluß der Bänder den Einfluß der Störstellen verdrängt, liegt um so höher, je größer die Störstellenkonzentration ist.

\section{Die Berechnung der Grenzenergie}

Wie einleitend gezeigt wurde, bedeutet die $\mathrm{Be}$ rechnung der Grenzenergie die Auswertung des In-

\footnotetext{
7 Über Wege zur allgemeinen Berechinung der Grenzenergie bzw. der daraus hervorgehenden Konzentration freier Elektronen und Defektelektronen siehe den Bericht von W.S ch ot tky, Z. Elektrochem. angew. physik. Chem. 45, 33 [1939], insbes. $\$ 12$. Dort ist für die Grenzenergie die in der physikalischen Chemie übliche Bezeichnung „chemisches Potential“ gebraucht.
}

tegrals (2), wobei wir uns auf das F-Band und GBand beschränken können ${ }^{7}$. Man stellt eine „Elektronenbilanz" auf und bestimmt $\zeta$ so, daß

$$
\int_{\mathrm{F}, \mathrm{G}} \frac{g \cdot D(E)}{1+e^{(E-\zeta) / k T}} d E=N
$$

wird, wobei die rechte Seite die Zahl der Elektronen bedeutet, deren Energie zwischen dem unteren Rand des G-Bandes und dem oberen Rand des F-Bandes liegt. Für ein Niveau bestimmter Energie tritt an Stelle eines Beitrages zu dem Integral ein Summand von der Form

$$
\frac{N_{i}}{1+e^{\left(E_{i}-\zeta\right) / k T}}
$$

wobei $N_{i}$ die Anzahl der Störterme pro Volumeneinheit und $E_{i}$ ihre Lage auf der Energieskala angibt.

Die Auswertung dieser Bilanz ist numerisch immer möglich. Folgende typische Vereinfachungen sind von Interesse:

Terme, die über einen breiten Energiebereich verteilt sind, können näherungsweise durch ein diskretes Niveau ersetzt werden, wenn der Bereich mehrere $k T$ von $\zeta$ entfernt ist. Wenn z. B. zwischen $E_{0}$ und $E_{1}$, die beide mehrere $k T$ oberhalb $\zeta$ liegen sollen, Terme nach einer Verteilung $D(E)$ liegen, so werden sie besetzt sein von

$$
n_{\mathrm{H}}=\int_{E_{0}}^{E_{1}} \frac{\mathrm{g} \cdot D(E) d E}{1+e^{(E-\zeta) / k T}}
$$

Elektronen. $n_{\mathrm{H}}$ ist also die Konzentration der an Haftstellen gebundenen Elektronen. Da nach den gemachten Voraussetzungen im ganzen Integrationsbereich $E \gg \zeta$ ist, kann man näherungsweise schreiben:

$$
n_{\mathrm{H}}=g \cdot e^{\zeta / k T} \int_{E_{0}}^{E_{1}} D(E) \cdot e^{-E / k T} d E
$$

und wenn $E_{1}$ mehrere $k T$ größer ist als $E_{0}$, so kann man das Integral von $E_{0}$ bis $\infty$ erstrecken. Daraus folgt durch mehrfaches partielles Integrieren:

$n_{\mathrm{H}}=g \cdot k T \sum_{i=0}^{\infty}(k T)^{i}\left[\frac{d^{i} D}{(d E)^{i}}\right]_{E=E_{0}} \cdot e^{\left(\zeta-E_{0}\right) / k T}$

Das ist aber'gerade die Zahl der Elektronen, die einen mehrere $k T$ oberhalb $\zeta$ gelegenen Term der 
Energie $E_{0}$ und des statistischen Gewichtes

$$
N_{\mathrm{H}}=g \cdot k T \sum_{i=0}^{\infty}(k T)^{i}\left[\frac{d^{i} D}{\left(a E^{\prime}\right)^{i}}\right]_{E=E_{0}},
$$

besetzen, denn für $\left(E_{0}-\zeta\right) \gg k T$ wird ein Term des stat. Gewichtes $N_{\mathrm{H}}$ von $n_{\mathrm{H}}=N_{\mathrm{H}} \exp \left[-\left(E_{0}-\zeta\right) / k T\right]$ Elektronen besetzt sein.

Für das erlaubte Energieband gilt in der Nähe des Bandrandes $D(E)=4 \pi \sqrt{2} m^{3 / 2} E^{1 / 2} h^{-3}(m=$ scheinbare Elektronenmasse). Hiermit und mit $g=2$ liefert die Integration von (9) den bekannten Ausdruck für das Gewicht des Bandes

$$
N_{0}=2\left(2 \pi m k T h^{2}\right)^{3 / 2} .
$$

Hat man eine homogene Verteilung von Störtermen in einem gewissen Bereich, so läßt sich deren Beitrag zur Elektronenbilanz sofort angeben, da jetzt $D(E)=$ const ist. Seien zwischen $E_{0}$ und $E_{1}$ Terme der konstanten Dichte $D$, so werden diese besetzt von

$$
n_{\mathrm{H}}=\int_{E_{0}}^{E_{1}} \frac{g D d E}{1+e^{(E-\zeta) / k T}}
$$

Elektronen. Die Integration liefert nach elementarer Rechnung:

$$
\begin{aligned}
n_{\mathrm{H}}=g D\left\{\left(E_{1}-\right.\right. & \left.E_{0}\right)+k T \\
& \left.\cdot \ln \frac{1+e^{\left(E_{0}-\zeta\right) / k T}}{1+e^{\left(E_{1}-\zeta\right) / k T}}\right\} .
\end{aligned}
$$

In engem Zusammenhang mit der Temperaturabhängigkeit der' Grenzenergie steht die Temperaturabhängigkeit der Leitfähigkeit eines Halbleiters. Hierfür macht man häufig den Ansatz

$$
\sigma=a e^{-b / T} .
$$

Er wird am einfachsten gedeutet durch eine Anzahl freier Elektronen

$$
n_{\mathrm{F}}=N_{0} e^{-\Delta E / k T} .
$$

Es wurde der Versuch gemacht, $N_{0}$ und $(E-\zeta)(,, a-$ Konstanten“ und „b-Konstanten“) als Materialeigenschaft zu messen ${ }^{8}$. Das Ergebnis ist für die $b$-Konstanten eine ziemlich beträchtliche Streuung um einen Mittelwert, während die $a$-Konstanten überhaupt

Z. B. E. Engelhard, Ann. Physik 17, 501 [1933]; L. Eis enmann, ebenda 38, 121 [1940]; B. Gudden, Ergebn. exakt. Naturwiss. XIII, 233 [1934]. keine Aussage zulassen, sondern bei demselben Halbleiter um Zehnerpotenzen streuen. Dieses Ergebnis ist jetzt verständlich: solange $\zeta$ unabhängig von $T$ ist, wären wirklich eine $a$-Konstante und eine $b$-Konstante zu erwarten. Nähert man $\zeta$ durch einen linearen Ansatz $\zeta=\zeta_{0}+\alpha T$, so wird

$$
n_{\mathrm{F}}=N_{0} e^{\alpha / k} e^{-\left(E-\zeta_{n}\right) / k \cdot T} .
$$

Die $a$-Konstante ist jetzt bereits um einen Faktor $e^{\alpha / k}$ verändert, während als $b$-Konstante tatsächlich $\left(E-\zeta_{0}\right) / k$ temperaturunabhängig eingeht. Entwickelt man also $\zeta$ nach steigenden Potenzen von $T$, so wird die $a$-Konstante bereits durch das Glied 1. Ordnung, die $b$-Konstante dagegen erst durch das quadratische Glied beeinflußt.

\section{Beispiele}

Hat man $\zeta$ berechnet, so ist die Zahl der Leitungselektronen

$$
n_{\mathrm{F}}=\frac{N_{0}}{1+e^{(E-\zeta) / k T}}
$$

und die Zahl der Leitungslöcher

$$
n_{\mathrm{L}}=\frac{N_{0}}{1+e^{\zeta / k T}} .
$$

$n_{\mathrm{F}}$ und $n_{\mathrm{L}}$ sind entscheidend für die Leitfähigkeit und den Halleffekt. Sie ändern sich mit der Konzentration der Störstellen und der Temperatur so stark, daß daneben die Beweglichkeit als eine Konstante angesehen werden kann. Sehen wir auch noch von der Verschiedenheit der Beweglichkeit für Elektronen und Löcher ab, so wird

die Leitfähigkeit $\sigma-\left(n_{\mathrm{F}}+n_{\mathrm{L}}\right)$

und die Hall-Konstante

$$
l i-\frac{n_{\mathrm{L}}-n_{\mathrm{F}}}{\left(n_{\mathrm{L}}+n_{\mathrm{F}}\right)^{2}} .
$$

Bewegt sich $\zeta$ von der Bandmitte weg nach oben, so steigt $n_{\mathrm{F}}$, bewegt es sich nach unten, so steigt $n_{\mathrm{L}}$ exponentiell an. Wenn die Elektronen gerade ausreichen, um die Störterme bis zur Mitte des Bandabstandes zu besetzen, so liegt $\zeta$ in der Mitte zwischen den Bändern, die Leitfähigkeit hat ein Minimum, und die Leitung wird gleichmäßig durch freie Elektronen und freie Löcher besorgt. $R$ ist in diesem Falle null, weil der Zähler verschwindet, hat aber in unmittelbarer Nähe Extremwerte. 
Für die Abb. 2-7 wurde die Grenzenergie aus der Elektronenbilanz nach Abschn. 2 berechnet, daraus die Zahl der freien Elektronen $n_{\mathrm{F}}$ und der Löcher $n_{\mathrm{L}}$ bestimmt und die rechten Seiten von (20) und (21) als Maße für die Leitfähigkeit und die Hallkonstante in Abhängigkeit von Störstellenkonzentration und Temperatur aufgetragen.

$\mathrm{Als}$ besonders lehrreiche Beispiele sind im folgenden 2 Stoffe gewählt, die sowohl Defekt- als Überschußleitung zeigen können, d. h. bei denen $\zeta$ sowohl unterhalb wie oberhalb der Mitte zwischen den Bän-

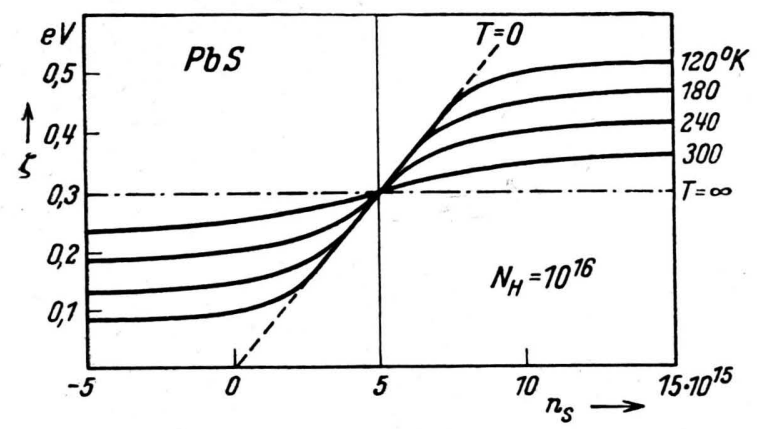

Abb. 2. Abhängigkeit der Grenzenergie von der Störstellenkonzentration für $\mathrm{PbS}$ bei einer Haftstellenkonzentration von $10^{16} \mathrm{~cm}^{-3}$.

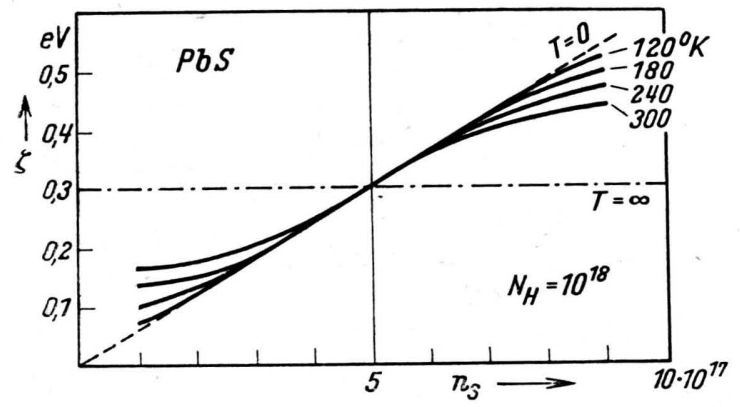

Abb. 3. Abhärgigkeit der Grenzenergie von der Störstellenkonzentration für $\mathrm{PbS}$ bei einer Haftstellenkonzentration von $10^{18} \mathrm{~cm}^{-3}$.

dern liegen kann: $\mathrm{PbS}$ und $\mathrm{Cu}_{2} \mathrm{O}$. PbS ist je nach der Herstellung Überschuß- oder Defektleiter, ändert also seinen Leitungsmechanismus in Abhängigkeit von der Störstellenkonzentration. Kupferoxydul ist bei Zimmertemperatur immer ein Defektleiter, über $500^{\circ} \mathrm{C}$ ein Überschußleiter. Es ändert also seinen Leitungsmechanismus als Funktion der Temperatur.

a) $\mathrm{Pb} \mathrm{S}$. Beim PbS haben wir einen Bandabstand von etwa $0,6 \mathrm{eV}$ anzunehmen, entsprechend einer lichtelektrischen Grenze von etwas über $2 \mu$. Wie das Experiment zeigt und die Abbildungen verständlich machen, genügen sehr kleine Änderungen in der

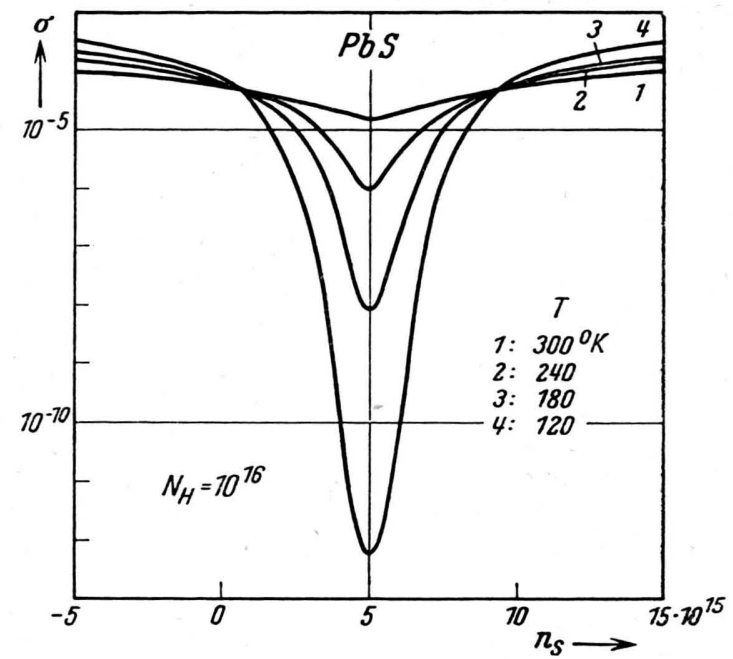

Abb. 4. Abhängigkeit der Leitfähigkeit von der Störelektronenkonzentration für $\mathrm{PbS}$ bei einer Haftstellenkonzentration von $10^{16} \mathrm{~cm}^{-3}$.

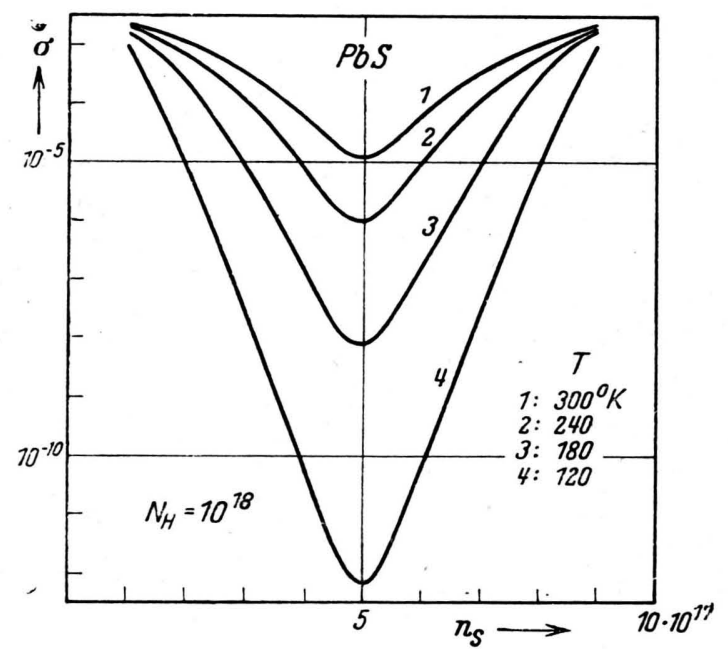

Abb. 5. Abhängigkeit der Leitfähigkeit von der Störelektronenkonzentration für $\mathrm{PbS}$ bei einer Haftstellenkonzentration von $10^{18} \mathrm{~cm}^{-3}$.

Zusammensetzung, um Betrag und Art der Leitfähigkeit bzw. Betrag und Vorzeichen des Halleffektes zu ändern. Wir rechnen deshalb mit einer Konzentration der Störterme von $10^{16}$ und $10^{18} \mathrm{im}$ $\mathrm{cm}^{3}$ (das entspricht Verunreinigungen von $10^{-4}$ und $\left.10^{-2} \%\right)$. Sie seien homogen über den Energiebereich zwischen den Bändern verteilt. Dann variieren wir die Zahl der überschüssigen Elektronen in einem entsprechenden Bereich und erhalten so die Abb. 2 und 3.

Ganz allgemein steigt die Grenzenergie mit zunehmender Zahl der Störelektronen $n_{\mathrm{S}}$ an. Für 
$T=0^{\circ} \mathrm{K}$ wäre der Anstieg eine Gerade, die durch die Punkte $n_{\mathrm{S}}=0, \zeta=0$ und $n_{\mathrm{s}}=N_{\mathrm{H}}, \zeta=0,6 \mathrm{eV}$ (Bandabstand) festgelegt ist. Mit wachsender Temperatur weichen die Kurven von der Geraden immer mehr ab. Sie fächern um so stärker auf, je geringer die Konzentration der Haftstellen ist. Bei sehr hohen Temperaturen würde $\zeta$ in der Mitte zwischen den Bändern liegen, die Kurve also zu einer Parallelen zur Abszissenachse entarten.

Um diese Abhängigkeiten mit dem Experiment zu vergleichen, muß man aus den Grenzenergien die Leitfähigkeiten berechnen. Das Ergebnis zeigen die Abb. 4 und 5.

Die berechneten Ergebnisse entsprechen den Kurven, die $\mathrm{H}$ in te $\mathrm{n}$ b e r g e ${ }^{9}$ an aufgedampften $\mathrm{PbS}$ Schichten gemessen hat. Dabei ist zu beachten, daß bei Hintenberger die Temperaturen der Temperung aufgetragen sind. Die Kurven sind nicht wie die theoretischen zur Mitte symmetrisch. Das bedeutet nur, daß die Geschwindigkeit, mit der der Schwefel ausgetempert wird, stärker als proportional der Temperungstemperatur wächst.

Hintenberger und andere Autoren, die ähnliche Messungen gemacht haben, nehmen stillschweigend an, daß das Minimum der Leitfähigkeit den Punkt der genauen stöchiometrischen Zusammensetzung angibt. Das ist nach unseren Überlegungen nicht der Fall. Vielmehr bedeutet das Minimum, daß gerade halb so viel überschüssige Elektronen wie Terme zwischen den Bändern vorhanden sind.

In Abb. 6 ist die Abhängigkeit der Leitfähigkeit und der Hallkonstanten von der Konzentration der Störelektronen bei einer Temperatur von $300^{\circ} \mathrm{K}$ und einer Haftstellenkonzentration von $10^{18} \mathrm{im} \mathrm{cm}^{3}$ aufgetragen. Es zeigt sich, daß die Hallkonstante in der Nähe des Leitfähigkeitsminimums zu einem sehr hohen Maximum ansteigt, im Leitfähigkeitsminimum durch Null geht und unmittelbar jenseits einem sehr hohen negativen Wert zustrebt. Das Maximum bzw. Minimum der Hallkonstanten liegt in unmittelbarer Nähe des Leitfähigkeitsminimums, und der Umschlag erfolgt innerhalb eines so engen Bereiches, daß nur bei sehr genauem Erreichen des Leitfähigkeitsminimums ein Meßpunkt auf dem abstürzenden Teil der Halleffektkurve gefunden wird. Praktisch wird man also bei sinkender Leitfähigkeit stets ein Ansteigen im Absolutbetrag des Halleffekts erwarten müssen, wie es auch die von $\mathrm{H}$ intenberg e ${ }^{10}$ gemessene Kurve zeigt.

${ }^{9}$ H. H inten berger, Z. Physik 119, 13 [1942], Abb. 9 .
In Abb. 6 bedeutet wachsender Abszissenwert $n_{8}$ eine Zunahme der elektronegativen Komponente, also des Schwefels. Für den Vergleich mit dem Experiment ist wieder $\mathrm{zu}$ beachten, daß auf der Abszissenachse der Hintenbergerschen Kurve nicht gleichmäßige Zunahmen des Schwefelgehaltes auf-

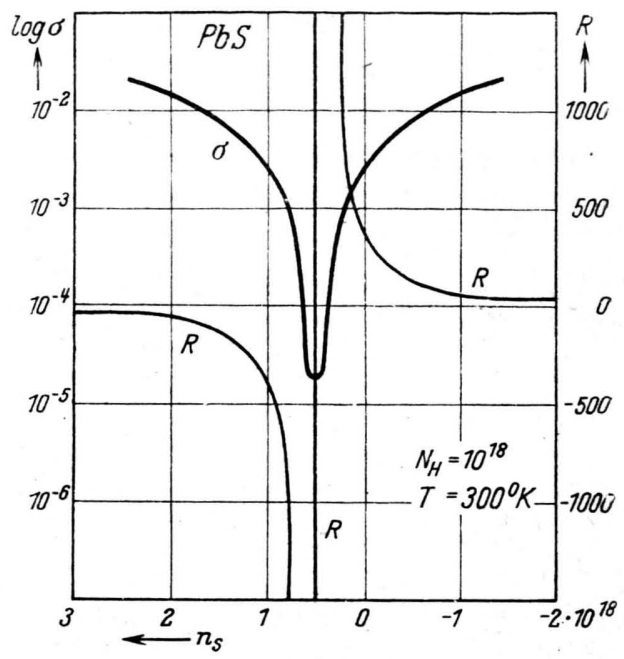

Abb. 6. Abhängigkeit der Leitfähigkeit und des HallEffektes von der Störelektronenkonzentration für $\mathrm{PbS}$ bei einer Haftstellenkonzentration von $10^{18} \mathrm{~cm}^{-3}$.

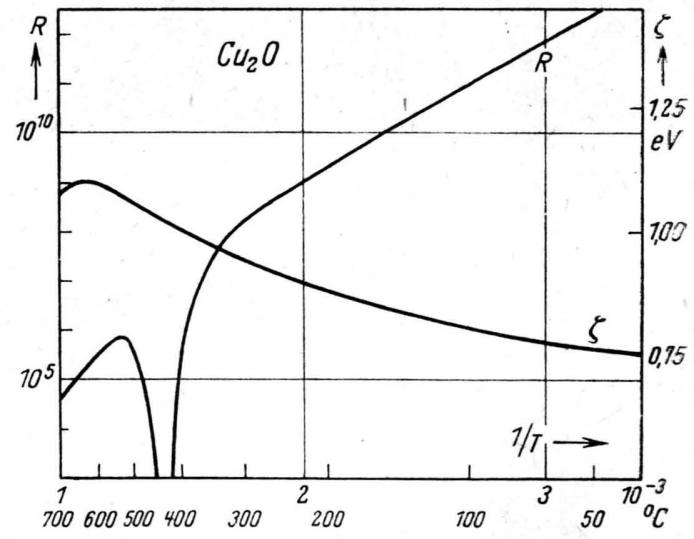

Abb. 7. Temperaturabhängigkeit der Grenzenergie und der Hall-Konstanten für $\mathrm{Cu}_{2} \mathrm{O}$.

getragen werden können. Wenn man das berücksichtigt, stimmen die Kurven für die Leitfähigkeit befriedigend überein. Bei den experimentellen Halleffektkurven sind die beiden Äste geradlinig verbunden, weil keine Zwischenwerte vorliegen.

b) $\mathrm{Cu}_{2} \mathrm{O}$. Beim Kupferoxydul finden $\mathrm{W}$ a i b e l und $\mathrm{Schottky}{ }^{11}$ zwischen 400 und $500^{\circ} \mathrm{C}$ einen Um-

${ }^{10} \mathrm{H}$. H in te $\mathrm{n}$ berger ${ }^{9}$, Abb. 12.

${ }^{11}$ Waibel u. S chottky, Physik. Z. 34, 858 [1933]. 
schlag des Hall-Effekts vom anomalen zum normalen Vorzeichen. Das bedeutet nach unseren Überlegungen, daß sich die Grenze $\zeta$ über die Mitte zwischen den Bändern hinwegbewegt. Für die numerische Berechnung wurde für den Bandabstand (nach der Grenze des inneren lichtelektrischen Effektes bei etwa $0,6 \mu) 2 \mathrm{eV}$ angenommen. Eine schwache lichtelektrische Empfindlichkeit findet sich bei $2 \mu$, entsprechend etwa $0,6 \mathrm{eV}$. Wir nehmen an, daß dieser energetische Abstand ein Störniveau bedeutet, und setzen ihn, da wir über eine genaue Termverteilung nichts wissen, versuchsweise als Grenze eines Gebietes homogen verteilter Störniveaus an.

Mit diesen Annahmen und passend gewählten Störstellenkonzentrationen $\left(N_{\mathrm{H}}-n_{\mathrm{s}}=2 \cdot 10^{15}\right)$ erhält man eine Abhängigkeit der Grenzenergie und des Hall-Effekts von der Temperatur, wie sie Abb. 7 zeigt und der experimentell gefundenen Kurve von $\mathrm{W}$ a i b e l und S chot tk $\mathrm{y}^{12}$ weitgehend entspricht.

\section{Zus ammenfass ung}

Es werden die Vorstellungen des Wilsonschen Halbleitermodells numerisch durchgeführt; dabei wird gezeigt, daß bei den Halbleitern die Fermische Grenzenergie nicht wie bei den Metallen als eine Konstante anzusehen ist,

12. Wa ibel u. S chot tky ${ }^{11}$, S. 859, Abb. 1. - Die untere der beiden dort gezeigten Kurven wurde von den Verff. später zurückgezogen (Physik. Z, 36, 912 [1936]). sondern daß sie durch Störstellenkonzentration und Temperatur so großen Veränderungen unterworfen ist, daß diese das elektrische Verhalten des Halbleiters charakterisieren. Im einzelnen:

1. Es wird die Temperaturabhängigkeit der Grenzenergie diskutiert.

2. Als Sonderfall zu 1. wird hergeleitet, daß die Grenzenergie temperaturunabhängig ist, wenn die Elektronenterme symmetrisch um die Grenzenergie verteilt sind.

3. An einem Modellhalbleiter werden die typischen Fälle der Abhängigkeit der Grenzenergie von Temperatur und Störstellenkonzentration dargestellt.

4. Aus 3. ergeben sich die Gültigkeitsgrenzen des Ansatzes für die Leitfähigkeit eines Halbleiters $\sigma=a e^{-b / T}$; dabei wird erklärt, warum die " $a$-Konstanten“ stärker streuen als die „b-Konstanten“.

5. Am Beispiel des $\mathrm{PbS}$ und des $\mathrm{Cu}_{2} \mathrm{O}$ wird die Grenzenergie für verschiedene Temperaturen und Störstellenkonzentrationen berechnet und die daraus folgenden Abhängigkeiten der Leitfähigkeit und des Hall-Effekts mit den experimentell gefundenen Kurven verglichen. Es zeigt sich gute Úbereinstimmung ${ }^{13}$.

Hrn. Prof. Schottky danke ich aufrichtig für wertvolle briefliche Hinweise.

13 Anm. b. d. Korr.: Nach neueren Messungen (L. Genzel und H. M ü s er, Z. Physik, im Druck) muß der Bandabstand für das PbS-Grundgitter zu etwa $3,9 \mathrm{eV}$ angenommen werden, während der in der vorliegenden Arbeit eingesetzte Abstand von etwa $0,6 \mathrm{eV}$ einem Störniveau zuzuordnen ist. Hierdurch werden die zahlenmäßigen Ergebnisse für das PbS-Beispiel modifiziert.

\title{
Beobachtungen an den Raman-Linien binärer und ternärer Gemische von Aceton, Methylalkohol und Sctwefelkohlenstoff
}

\begin{abstract}
Von Maria Joerges und Alexander Nikuradse
Aus dem Institut für Elektronen- und Ionenforschung der Technischen Hochschule München

(Z. Naturforschg. 5a, 25-34 [1950]; eingegangen am 21. April 1949)

In binären und ternären Gemischen von Aceton, Methylalkohol und Schwefelkohlenstoff werden Verschiebungen einiger Raman-Schwingungslinien gegen die Frequenzen der Reinsubstanzen beobachtet. Diese Verschiebungen zeigen, in welcher Weise Aceton- und Methanolmoleküle im Gemisch gekoppelt sind. Die Beobachtung der Intensitätsverhältnisse spezieller Linien gestattet Aussagen über das relative Streuvermögen, dessen Verhalten auf eine Änderung der Polarisierbarkeit infolge des durch Beimischungen beeinflußten Bindungszustandes der Moleküle zurückgeführt werden muß.
\end{abstract}

$\mathrm{D}$ er Smekal-Raman-Effekt gestattet nicht allein Aussagen über Molekülstrukturen und Bindungskräfte zu machen, er erlaubt z. B. neben der Analyse von Systemen aus verschiedenen Molekülsorten auch Aussagen über zwischenmolekulare Kräfte und deren Rückwirkung auf innermolekulare Kräfte. Da an Flüssigkeitsgemischen dieselben Streulinien gefunden wurden, die für die Gemischkomponenten charakteristisch sind, war man längere Zeit hindurch der Meinung, daß diese auch im Gemisch unabhängig voneinander erscheinen.

Bald zeigte sich jedoch, daß diese Additivität offenbar nur eine erste Näherung ist, da man sowohl bei Änderungen der Aggregatzustände der Reinsubstan- 